\title{
Rendimento de tambaqui em diferentes métodos de filetagem
}

\author{
Tambaqui yield in different filleting methods \\ Rendimiento de tambaqui en diferentes métodos de fileteado
}

Recebido: 12/03/2021 | Revisado: 21/03/2021 | Aceito: 25/03/2021 | Publicado: 02/04/2021

\author{
Amélia Maria Lima Garcia \\ ORCID: https://orcid.org/0000-0002-0111-9279 \\ Instituto Federal de Educação, Ciência e Tecnologia do Acre, Brasil \\ E-mail: amelia.garcial@ifac.edu.br \\ Hévea Monteiro Maciel \\ ORCID: https://orcid.org/0000-0003-0342-2826 \\ Instituto Federal de Educação, Ciência e Tecnologia do Acre, Brasil \\ Programa de Recursos Pesqueiros e Engenharia de Pesca, Brasil \\ E-mail: hevea.maciel@ifac.edu.br
}

\begin{abstract}
Resumo
O objetivo foi avaliar o rendimento de filé de tambaqui submetido a diferentes métodos de filetagem. O delineamento experimental foi inteiramente casualizado, com três métodos de filetagem e dezenove repetições. Os tratamentos foram baseados na retirada ou não da cabeça e nadadeiras do peixe antes do processo de filetagem e na forma de remover a pele, antes ou depois da remoção do filé. Os peixes apresentaram peso médio de 2620,81 g, comprimento total de 50,17 cm, comprimento padrão de 40,26 cm, largura do corpo de 19,32 cm. Os métodos de filetagem no tambaqui influenciaram significativamente $(\mathrm{P}<0,05)$ o peso do filé sem pele, filé com pele, pele bruta, pele limpa, carcaça, cabeça, resíduos totais. O método de filetagem PIVF apresentou maior peso do filé sem pele, filé com pele, pele bruta, pele limpa, carcaça, resíduos totais, mas não diferiu significativamente do método de filetagem PDEF. Os métodos de filetagem avaliados não influenciaram significativamente $(\mathrm{P}>0,05)$ o rendimento de filé sem pele, filé com pele, pele bruta, pele limpa, carcaça e dos resíduos totais. O método de filetagem com PIVF é o recomendado para a indústria de pescado para o aproveitamento de resíduos, pois apresenta maior volume de resíduos com potencial de matéria-prima de baixo custo para geração de novos produtos. Já o método de filetagem PDEN é o mais indicado quando a indústria de processamento não destina os resíduos para reaproveitamento.
\end{abstract}

Palavras-chave: Colossoma macropomum; Filé; Processamento de pescado.

\begin{abstract}
The objective was to evaluate the yield of tambaqui fillet submitted to different filleting methods. The experimental design was completely randomized, with three filleting methods and nineteen repetitions. The treatments were based on the removal or not of the head and fins of the fish before the filleting process and on the way to remove the skin, before or after the removal of the fillet. The tambaqui had an average weight of $2620.81 \mathrm{~g}$, total length of $50.17 \mathrm{~cm}$, standard length of $40.26 \mathrm{~cm}$, body width of $19.32 \mathrm{~cm}$. The filleting methods in tambaqui significantly influenced (P $<0.05)$ the weight of the skinless fillet, skinned fillet, raw skin, clean skin, carcass, head, total residues. The PIVF filleting method showed greater weight of the skinless fillet, skinned fillet, raw skin, clean skin, carcass, total residues, but did not differ significantly from the PDEF filleting method. The filleting methods evaluated did not significantly influence $(\mathrm{P}>0.05)$ the yield of skinless fillet, skinned fillet, raw skin, clean skin, carcass and total waste. The filleting method with PIVF is recommended for the fish industry for the use of residues, as it presents a greater volume of residues with low-cost raw material potential for the generation of new products. The PDEN filleting method is the most suitable when the processing industry does not allocate waste for reuse.
\end{abstract}

Keywords: Colossoma macropomum; Fillet; Fish processing.

\section{Resumen}

El objetivo fue evaluar el rendimiento del filete de tambaqui sometido a diferentes métodos de fileteado. El diseño experimental fue completamente al azar, con tres métodos de fileteado y diecinueve repeticiones. Los tratamientos se basaron en la remoción o no de la cabeza y aletas del pescado antes del proceso de fileteado y en la forma de remover la piel, antes o después de la remoción del filete. Los peces tenían un peso promedio de $2620,81 \mathrm{~g}$, longitud total de $50,17 \mathrm{~cm}$, longitud estándar de 40,26 cm, ancho corporal de 19,32 cm. Los métodos de fileteado en tambaqui influyeron significativamente $(\mathrm{P}<0.05)$ en el peso del filete sin piel, filete sin piel, piel cruda, piel limpia, canal, cabeza, residuos totales. El método de fileteado PIVF mostró mayor peso del filete sin piel, filete sin piel, piel cruda, piel limpia, canal, residuos totales, pero no difirió significativamente del método de fileteado PDEF. Los métodos de fileteado evaluados no influyeron significativamente $(\mathrm{P}>0.05)$ en el rendimiento de filete sin piel, filete sin piel, piel cruda, piel limpia, canal y desperdicio total. El método de fileteado con PIVF es recomendado para la industria 
pesquera para el aprovechamiento de residuos, ya que presenta un mayor volumen de residuos con potencial de materia prima de bajo costo para la generación de nuevos productos. El método de fileteado PDEN es el más adecuado cuando la industria de procesamiento no asigna residuos para su reutilización.

Palabras clave: Colossoma macropomum; Filete; Procesamiento de pescado.

\section{Introdução}

A piscicultura brasileira gerou no ano de 2014, o PIB de $\mathrm{R} \$ 5$ bilhões, mobilizou 800 mil profissionais e proporcionou 3,5 milhões de empregos diretos e indiretos (Brasil, 2014). Produziu em 2018, o total de 519,3 mil toneladas, aumentando de 3,4\% em relação ao ano anterior, gerando o valor de produção para a atividade de $\mathrm{R} \$ 3,3$ bilhões (IBGE, 2018). No Brasil, em 2013, o consumo de pescado foi de 19,7 Kg por pessoa (FAO, 2016), consequentemente, o sistema intensivo de cultivo de peixes teve que crescer rapidamente para aumentar a produtividade e atender as exigências do consumidor.

Dentre as espécies mais comercializadas da região amazônica está o tambaqui (Colossoma macropomum Cuvier 1818) (Nóbrega et al., 2020). É a segunda espécie de água doce mais produzida e a primeira nativa do Brasil, representando $19,7 \%$ do total da piscicultura brasileira, na qual a região Norte é responsável por 73,1\% do total de 102,6 mil toneladas da espécie no Brasil (IBGE, 2018). O tambaqui tem alta aceitação da carne no mercado consumidor devido ao excelente sabor da carne (Costa et al., 2012), sendo muito produzido principalmente pelo seu rápido ganho de peso, podendo render o produtor um lucro de 108\% (Izel \& Melo, 2004). O valor nutritivo e os preços dos peixes dependem da composição química, textura da carne, rendimento e fatores relacionados aos métodos de captura e beneficiamento (Lima et al., 2012). Segundo Dantas Filho et al., (2021) o valor calórico e teor proteico não tem correlação com o preço do quilo do pescado para os diferentes cortes comerciais, inferindo que os fatores sensoriais, mercadológicos e culturais são responsáveis pelas variações de preço.

O rendimento do processamento é dependente de diversos fatores e variando entre espécies, dentro da mesma espécie dependendo do tempo de cultivo e do peso de abate. O cultivo de tambaqui no período de 107, 137 e 167 dias proporciona variações entre o rendimento dos cortes, sendo que quanto maior o tempo de cultivo, maior rendimento de rendimento do peixe sem cabeça, pele e nadadeiras e rendimento do filé (Fernandes et al., 2018). O tambaqui com diferentes pesos de abate causa variações no rendimento de filé e vísceras (De Lima et al., 2018), rendimento do peixe eviscerado e de tronco limpo (Cirne et al., 2019). Em relação ao valor calórico em função da composição centesimal do tambaqui, a classe de peso de 2,41 a 3,5 kg é a categoria mais eficiente para produção e comercialização (Dantas Filho et al., 2021).

Outro fator importante que influencia o rendimento do processamento são as máquinas filetadoras ou da destreza manual do operador (De Faria et al., 2003), que consequentemente geram diferente volume de resíduos. O uso dos resíduos da industrialização do pescado na elaboração de produtos permite a transformação de resíduos em alimentos de alta qualidade nutricional a baixo custo e minimiza a poluição ambiental (Batalha et al., 2017). Entre os produtos elaborados com a reutilização dos resíduos estão os óleos, farinhas, silagens de pescado, que são formas de controlar os impactos gerados pelos resíduos (De Lima, 2013). Também podem ser produzidas gelatinas a partir dos resíduos da filetagem de matrinxã e tambaqui (cabeça, espinhaço, nadadeiras, couro e escamas) com alto teor proteico (Da Silva et al., 2018).

Desta forma, pesquisas são necessárias para definir métodos de cortes, principalmente da filetagem para agregar valor comercial e diversificar o processamento das espécies nativas no Brasil (Pedroza Filho et al., 2014). Principalmente, que investiguem o potencial do tambaqui, quanto ao seu rendimento em diferentes métodos de processamento, pois informações essenciais para o desenvolvimento da cadeia produtiva do tambaqui, uma vez que, no Brasil não existe um padrão no método de filetagem do tambaqui. Assim, o objetivo do presente trabalho foi avaliar o rendimento de filé de tambaqui submetido a diferentes métodos de filetagem. 


\section{Metodologia}

O experimento foi um estudo realizado no laboratório de alimentos do Campus Rio Branco Avançado Baixada do Sol do Instituto Federal de Educação, Ciência e Tecnologia do Acre, localizado no município de Rio Branco. Foram utilizados 57 peixes tambaqui (Colossoma macropomum) provenientes de piscicultura localizada no município de Rio Branco - Acre.

O delineamento experimental foi inteiramente casualizado constituído de três tratamentos (métodos de filetagem) e dezenove repetições, sendo cada peixe considerado uma unidade experimental. Os tratamentos foram baseados na retirada ou não da cabeça e nadadeiras do peixe antes do processo de filetagem e na forma de remover a pele, antes ou depois da remoção do filé. Os tratamentos foram:

PIVF = No peixe inteiro e com vísceras foi retirado o filé com pele e, em seguida, a pele e o filé foram reparados com o auxílio de uma faca;

PDEF = No peixe decapitado e eviscerado foi retirado o filé com pele e, em seguida, a pele e o filé foram reparados com o auxílio de uma faca;

PDEN = No peixe decapitado e eviscerado foram removidas as nadadeiras (dorsal e anal), posteriormente será retirado o filé com pele e, em seguida, a pele e o filé foram reparados com o auxílio de uma faca.

Os animais foram submetidos a 24 horas de jejum antes da despesca e abatidos aleatoriamente e insensibilizados por choque térmico, por meio do contato com solução água-gelo e abatidos por anóxia. Os animais foram abatidos de manhã cedo pelo piscicultor e posteriormente foram entregues no laboratório do IFAC para realização das pesagens e demais procedimentos para obtenção de dados experimentais.

Ao chegar no laboratório foram realizadas avaliações biométricas com auxílio com auxílio de um ictiômetro (graduado em $\mathrm{cm}$ ) e um paquímetro (graduado $\mathrm{em} \mathrm{mm}$ ). Foram mensuradas comprimento total (CT), comprimento padrão (CP) - compreendendo entre a extremidade anterior da cabeça e o menor perímetro do pedúnculo caudal (inserção da nadadeira caudal) e largura do corpo (LC) - largura do corpo tomada na região do $1^{\circ}$ raio das nadadeiras dorsal.

Cada peixe foi submetido a pesagem para obtenção do peso corporal total (PCT), peso do filé sem pele (PFSP), peso do filé com pele (PFCP), peso da pele bruta = pele após a esfola, com escamas e restos de músculos (PPB), peso da pele limpa = pele após escamar e descarnar (PPL), peso da carcaça = peixe com cabeça, filé e eviscerada (PCAR), peso da cabeça $(\mathrm{PCAB})$, peso dos resíduos totais = vísceras com gordura, coluna vertebral, nadadeiras e pele bruta $($ PREST).

Após a pesagem, cada tambaqui foi filetado por uma única pessoa de acordo com cada tratamento. Nos métodos de filetagem onde a cabeça do peixe foi retirada foi aplicado o corte tipo reto ao comprimento do peixe. Considerando o peso corporal total de cada peixe foram calculados o rendimento de filé sem pele (RFSP), rendimento de filé com pele (RFCP), rendimento de pele bruta (RPB), rendimento de pele limpa (RPL), rendimento de carcaça (RCAR), rendimento de cabeça (RCAB) e o rendimento dos resíduos totais (RREST).

Os resultados foram submetidos à análise de variância (ANOVA) e as médias comparadas pelo teste de Tukey ao nível de 5\% de probabilidade. Utilizou-se o programa estatístico SAS ${ }^{\circledR}$ OnDemand for Academics, versão online.

\section{Resultados e Discussão}

Os peixes apresentaram peso médio de $2620,81 \mathrm{~g}$ e nas avaliações biométricas observou-se comprimento total (CT) de $50,17 \mathrm{~cm}$, comprimento padrão (CP) de 40,26 cm, largura do corpo (LC) de 19,32 cm.

O peso médio de abate na produção de tambaqui varia de acordo a demanda do nicho de mercado. Atualmente, o peso mínimo considerado bom para o abate do tambaqui é de $1,4 \mathrm{~kg}$. A indústria que é o destino da maioria da produção tambaqui requer peixes com peso médio de $\pm 2 \mathrm{~kg}$, contudo peixes acima de $\pm 5 \mathrm{~kg}$ tem a preferência dos consumidores. Para atender à 
demanda por animais de grande porte, o cultivo de fêmeas é o mais indicado, devido ao seu crescimento de capacidade mais rápido que os machos (Mello et al., 2015).

As curvas de crescimento do tambaqui sugerem que o peso corporal é a característica que antecede o desenvolvimento das demais características morfológicas (peso corporal, comprimento corporal, altura corporal e comprimento da cabeça), sugerindo essas são características de desenvolvimento tardio. O padrão de crescimento corporal do tambaqui indica que o peixe continua crescendo após atingir 1,4 kg ou após 630 dias de cultivo, indicadores comuns para abate (Rosa et al., 2018).

O comprimento total e comprimento padrão registrados nesta pesquisa são corroborados por De Lima et al. (2018), que na avaliação de medidas biométricas do tambaqui em função da categoria de peso observou que peixes abatidos acima de $2,0 \mathrm{~kg}$ apresentaram comprimento total e comprimento padrão de 46,8 e 37,7 cm, respectivamente. Do mesmo modo, 47,1 e $38,4 \mathrm{~cm}$ de comprimento total e comprimento padrão, respectivamente foi encontrado no tambaqui abatido entre 2,0 e $2,5 \mathrm{~kg}$ (Cirne et al., 2019).

Os métodos de filetagem no tambaqui influenciaram significativamente $(\mathrm{P}<0,05)$ o peso do filé sem pele, peso do filé com pele, peso da pele bruta, peso da pele limpa, peso da carcaça, peso da cabeça, peso dos resíduos totais (Tabela 1). O método de filetagem PIVF apresentou maior peso do filé sem pele, peso do filé com pele, peso da pele bruta, peso da pele limpa, peso da carcaça, peso dos resíduos totais, mas não diferiu significativamente do método de filetagem PDEF (Tabela 1). Entretanto, os métodos de filetagem PIVF e PDEF proporcionaram maior peso da cabeça. Diante dos resultados verifica-se que método de filetagem PIVF do tambaqui gera maior volume de resíduos durante o processamento.

Tabela 1 - Valores médios dos pesos dos cortes dos métodos de filetagem.

\begin{tabular}{lrrrrrr}
\hline \multirow{2}{*}{ Variáveis $^{1}$} & \multicolumn{3}{c}{ Métodos de filetagem $^{2}$} & \multirow{2}{*}{ Média (g) } & \multirow{2}{*}{ CV $^{3}(\%)$} & Valor-P \\
\cline { 2 - 4 } & \multicolumn{1}{c}{ PIVF } & PDEF & PDEN & & & \\
\hline PFSP (g) & $1089,89 \mathrm{~A}$ & $946,53 \mathrm{AB}$ & $822,95 \mathrm{~B}$ & 953,12 & 22,22 & 0,0013 \\
PFCP (g) & $1310,95 \mathrm{~A}$ & $1138,95 \mathrm{AB}$ & $996,00 \mathrm{~B}$ & 1148,63 & 20,60 & 0,0006 \\
PPB (g) & $198,84 \mathrm{~A}$ & $175,79 \mathrm{AB}$ & $158,32 \mathrm{~B}$ & 177,65 & 22,94 & 0,0128 \\
PPL (g) & $104,11 \mathrm{~A}$ & $920,00 \mathrm{AB}$ & $83,37 \mathrm{~B}$ & 93,16 & 28,31 & 0,0600 \\
PCAR (g) & $2631,05 \mathrm{~A}$ & $2335,68 \mathrm{AB}$ & $2064,63 \mathrm{~B}$ & 2343,79 & 19,93 & 0,0020 \\
PCAB (g) & $579,26 \mathrm{~A}$ & $455,79 \mathrm{~A}$ & $412,63 \mathrm{~B}$ & 482,56 & 18,58 & $<, 0001$ \\
PREST (g) & $1284,00 \mathrm{~A}$ & $1142,84 \mathrm{AB}$ & $973,47 \mathrm{~B}$ & 1133,44 & 22,89 & 0,0023 \\
\hline
\end{tabular}

Médias seguidas de letras diferentes na mesma linha diferem significativamente $(\mathrm{P}<0,05)$ pelo teste de Tukey. ${ }^{1}$ Variáveis: PFSP - Peso do filé sem pele. PFCP - peso do filé com pele. PPB - peso da pele bruta. PPL - peso da pele limpa. PCAR - peso da carcaça. PCAB - peso da cabeça. PREST - peso dos resíduos totais. ${ }^{2}$ Métodos de filetagem: PIVF - Peixe inteiro e com vísceras foi retirado o filé com pele e, em seguida, a pele e o filé foram reparados com o auxílio de uma faca. PDEF - Peixe decapitado e eviscerado foi retirado o filé com pele e, em seguida, a pele e o filé foram reparados com o auxílio de uma faca. PDEN Peixe decapitado e eviscerado foram removidas as nadadeiras (dorsal e anal), posteriormente será retirado o filé com pele e, em seguida, a pele e o filé foram reparados com o auxílio de uma faca. ${ }^{3} \mathrm{CV}=$ coeficiente de variação.

Fonte: Autoras.

Além de avaliar o rendimento das partes processadas de peixe para definir o valor econômico do produto, planejamento da produção e eficiência produtiva da empresa (Carneiro et al., 2004), é importante conhecer o volume dos resíduos gerados pela indústria de processamento. Os resíduos de processamento de pescado são matérias-primas de baixo custo e podem ser usadas para geração de novos produtos, no entanto, se o volume de resíduos gerados não for aproveitado, eles serão depositados na natureza causando alto impacto ambiental, e assim contaminar solos e bacias hídricas (Camargo \& Pouey, 2005).

O aproveitamento dos resíduos de pescado no Brasil ainda é escasso, sendo que a maioria desses resíduos é descartado pelas indústrias de beneficiamento de pescado devido, principalmente, à falta de reconhecimento deste recurso como matéria- 
prima e fonte para outros produtos (Nascimento et al., 2014). Entre as várias formas de reaproveitamento de resíduos de peixe, a compostagem é uma alternativa viável diante dos parâmetros físico-químicos avaliados ao final do processo de compostagem que servem para avaliar a eficácia do processo (Valente et al., 2016; Dos Santos Nascimento et al., 2018). Assim como a utilização dos resíduos de filetagem de tilápia na produção de patês com adição de óleo de orégano é uma alternativa ao aproveitamento de um resíduo da indústria de pescados com diminuição do impacto ambiental, assim como, uma alternativa para agregar valor aos produtos (Matiucci et al., 2021).

A pele de tambaqui pode ser utilizada como um resíduo de qualidade para o processo de curtimento e aplicação na indústria de confecção de artefatos em geral ou mesmo de calçados e vestuário. Além disso, a pele de tambaqui apresenta teor de proteína bruta 35,70 \% e os couros de tambaqui apresentam maior resistência à tração e ao rasgamento progressivo, comparada aos couros de pacu e tilápia do Nilo. Entretanto, a retirada da pele de tambaqui apresenta maior resistência quando comparada a retirada da pele de tilápia, assim sendo essa dificuldade é compensada, a princípio com o abate de animais com peso acima de $1500 \mathrm{~g}$, proporcionando peles de maior área útil de aproveitamento (Franco et al., 2013).

A farinha de pele de tambaqui que pode ser agregada a pães, torradas, bolachas, entre outros, visando aumentar o teor proteico destes produtos (De Sá Leitão \& Favacho, 2015). O farelo de silagem biológica produzido a partir da biomassa residual de tambaqui pode ser incluído em até $5 \%$ nas dietas de poedeiras comerciais, pois apresenta boa digestibilidade de nutriente, portanto, pode ser potencialmente utilizado como fonte alimentar de energia e proteína (Guimarães et al., 2019). Silagens biológicas de pintado e pirapitinga apresentam níveis de proteína bruta de cerca de $42 \%$, evidenciando seu potencial como substituto de farinha de pescado em rações animais (De Lima et al., 2020).

Os métodos de filetagem avaliados não influenciaram significativamente $(\mathrm{P}>0,05)$ o rendimento de filé sem pele, rendimento de filé com pele, rendimento de pele bruta, rendimento de pele limpa, rendimento de carcaça e rendimento dos resíduos totais (Tabela 2). Por outro lado, o rendimento de cabeça foi influenciado significativamente $(\mathrm{P}>0,05)$ pelo método de filetagem, sendo que o método PDEN resultou em maior rendimento de cabeça sem diferir significativamente do método PDEF.

Tabela 2 - Valores médios dos rendimentos dos cortes dos métodos de filetagem.

\begin{tabular}{lrrrrrr}
\hline Variáveis $^{1}$ & \multicolumn{3}{c}{ Métodos de filetagem $^{2}$} & & Média & CV \\
\cline { 2 - 4 } & PIVF & PDEF & PDEN & & Valor-P \\
\hline RFSP (\%) & 36,61 & 36,32 & 35,40 & 36,11 & 7,22 & 0,3344 \\
RFCP (\%) & 44,21 & 43,73 & 42,96 & 43,63 & 5,56 & 0,2873 \\
RPB (\%) & 6,89 & 6,86 & 6,65 & 6,80 & 15,84 & 0,7612 \\
RPL (\%) & 3,61 & 3,58 & 3,48 & 3,56 & 21,22 & 0,0051 \\
RCAR (\%) & 90,80 & 89,53 & 87,80 & 89,38 & 6,84 & 0,3248 \\
RCAB (\%) & $19,44 \mathrm{~B}$ & $18,04 \mathrm{AB}$ & $17,78 \mathrm{~A}$ & 18,42 & 9,94 & 0,0156 \\
RREST (\%) & 44,40 & 43,01 & 42,31 & 43,24 & 14,83 & 0,5960 \\
\hline
\end{tabular}

Médias seguidas de letras diferentes na mesma linha diferem significativamente $(\mathrm{P}<0,05)$ pelo teste de Tukey. ${ }^{1}$ Variáveis: $\mathrm{RFSP}$ - Rendimento do filé sem pele. RFCP - Rendimento do filé com pele. RPB - Rendimento da pele bruta. RPL - Rendimento da pele limpa. RCAR - Rendimento da carcaça. RCAB Rendimento da cabeça. RREST - Rendimento dos resíduos totais. ${ }^{2}$ Métodos de filetagem: PIVF - Peixe inteiro e com vísceras foi retirado o filé com pele e, em seguida, a pele e o filé foram reparados com o auxílio de uma faca. PDEF - Peixe decapitado e eviscerado foi retirado o filé com pele e, em seguida, a pele e o filé foram reparados com o auxílio de uma faca. PDEN - Peixe decapitado e eviscerado foram removidas as nadadeiras (dorsal e anal), posteriormente será retirado o filé com pele e, em seguida, a pele e o filé foram reparados com o auxílio de uma faca. ${ }^{3} \mathrm{CV}=$ coeficiente de variação.

Fonte: Autoras. 
Neste trabalho apresentou-se bom rendimento filé, sendo a variação de 35,40 a $36,61 \%$ e 42,96 a 44,21\%, para rendimento do filé sem pele e rendimento do filé com pele, respectivamente (Tabela 2) T. Estes resultados estão acima do encontrado na mesma espécie por Fernandes et al. (2018), que alcançaram rendimento com variação entre 31,5 e 32,6\%.

Os melhores rendimentos de filetagem na tilápia do Nilo são obtidos com o tipo de corte de cabeça contornado, entretanto, visando a comercialização apenas de filé e tronco limpo pode ser usado o corte oblíquo ou contornado (De Souza et al., 2000). O método de filetagem no qual se retira a pele do peixe inteiro e depois remove o filé proporciona melhor resultado de rendimento de filé e músculo hipaxial profundo e bom resultado de pele bruta e limpa e menor porcentagem de resíduos de filetagem (Souza, 2002).

Pode ser observado (Tabela 2) que o rendimento de carcaça com cabeça, rendimento da cabeça e rendimento da pele desta pesquisa foi superior ao encontrado por Cirne et al. (2019) que encontraram valores de 82,61\%, 22,11\% e 3,03\%, respectivamente para tambaqui com peso ao abate entre 2,0 a 2,5 kg. Os mesmos autores observaram rendimento das vísceras de $6,47 \%$ e rendimento do resíduo de $12,67 \%$.

Nesta pesquisa não observou a influência do método de filetagem sobre o rendimento de filé e demais cortes, entretanto essa influência ocorreu em outras espécies. Em tilápias do Nilo, o método de retirar a pele com alicate e depois o filé proporcionou maior rendimento de carcaça $(56,43 \%)$ e filé $(36,67 \%)$, mas menor rendimento de pele bruta $(5,32 \%)$ e pele limpa (3,14\%) (Souza et al., 1999). No matrinxã, o processo de filetagem utilizando diferentes cortes de cabeça mostrou que o corte oblíquo da cabeça é o mais indicado para o processamento e comercialização, pois apresentou maior rendimento de filé $(37,23 \%)$ (Gomiero et al., 2003).

Peixes fusiformes apresentam altos rendimentos (> 54\%) devido à massa muscular cilíndrica, enquanto peixes compridos estão entre as espécies de rendimentos mais baixos (inferiores a 42\%), como no caso da tilápia (Contreras, 1994). Considerando as características morfológicas, os peixes de cabeça grande e comprimida proporcionam baixos rendimentos de filé, como para a tilápia do Nilo, e possuindo cabeça pequena, como o pacu, o rendimento atinge valores mais altos, evidenciando a existência de uma relação inversa entre tamanho da cabeça e rendimento de filé (De Faria et al., 2003).

O rendimento de filé de tilápia do Nilo foi em torno de 32,2\%, enquanto o rendimento do filé de pacu, 52,7\% (Souza e Vargas, 2001). O pacu (Piaractus mesopotamicus) destinado ao processo de filetagem deve ser abatido, preferencialmente, com peso entre 600 a $1285 \mathrm{~g}$, pois proporciona maior rendimento de filé (32,65\%), no entanto, peixes para comercialização na forma de carcaça ou inteiros eviscerados podem ser abatidos com pesos menores (Basso \& Silva, 2011).

\section{Conclusão}

O método de filetagem do tambaqui realizado com peixe inteiro e com vísceras para retirada do filé com pele e, em seguida, a pele e o filé com o auxílio de faca é o mais recomendado para a indústria de pescado quando ela destina os resíduos para reaproveitamento, pois é o método que não apresenta diferenças de rendimentos de filé e corte, mas apresenta maior volume de resíduos com potencial de matéria-prima de baixo custo para geração de novos produtos. Quando indústria de processamento não destina os resíduos para reaproveitamento o método mais indicado é quando a filetagem do tambaqui é realizada no peixe decapitado e eviscerado para remoção das nadadeiras (dorsal e anal) e posterior retirada do filé com pele, da pele e o filé com o auxílio de faca.

Neste sentido, recomenda-se outras investigações em relação a diferentes métodos de filetagem, que levem em consideração a categoria de peso e destreza do filetador empregado para cada método. 


\section{Referências}

Basso, L., Ferreira, M. W., \& Silva, A. R. (2011). Efeito do peso ao abate nos rendimentos dos processamentos do pacu (Piaractus mesopotamicus). Arquivo Brasileiro de Medicina Veterinária e Zootecnia, 63(5), 1260-1262. http://dx.doi.org/10.1590/S0102-09352011000500033.

Batalha, O. D. S., Alfaia, S. S., Cruz, F. G. G., Jesus, R. S. D., Rufino, J. P. F., \& Costa, V. R. (2017). Digestibility and physico-chemical characteristics of acid silage meal made of pirarucu waste in diets for commercial laying hens. Acta Scientiarum. Animal Sciences, 39(3), 251-257. http://dx.doi.org/10.4025/actascianimsci.v39i3.35112.

Brasil, A. C. E. E. (2014). $1^{\circ}$ Anuário Brasileiro da Pesca e Aquicultura. Rio de Janeiro.

Camargo, S. D., \& Pouey, J. L. O. F. (2005). Aquicultura-um mercado em expansão. Revista Brasileira de Agrociência, 11(4), $393-396$.

Carneiro, P. C. F., Mikos, J. D., Bendhack, F., \& Ignácio, S. A. (2004). Processamento do jundiá Rhamdia quelen: rendimento de carcaça. Revista Acadêmica Ciência Animal, 2(3), 11-17. http://dx.doi.org/10.7213/cienciaanimal.v2i3.15082.

Cirne, L. G. A., da Silva, J. R., de Souza, W. S., Brito, P. F., FELTRAN, R. D. B., Pereira, S. L. A., ... \& de Melo, D. R. (2019). Características morfométricas e da carcaça de tambaqui abatidos com diferentes pesos. Embrapa Roraima-Artigo em periódico indexado (ALICE).

Contreras, E. (1994). Bioquímica de Pescados e Derivados, Editora FUNEP.

Costa, G. D. M., Ortis, R. C., Lima, M. G. D., Casals, J. B., Lima, A. R. D., \& Kfoury Jr, J. R. (2012). Estrutura morfológica do fígado de tambaqui Colossoma macropomum (Cuvier, 1818). Pesquisa Veterinária Brasileira, 32(9), 947-950. https://doi.org/10.1590/S0100-736X2012000900022.

Dantas Filho, J. V., Cavali, J., Nunes, C. T., Nóbrega, B. A., da Fonseca Gasparini, L. R., de Souza, M. L. R., \& Pontuschka, R. B. (2021). Composición aproximada, valor calórico y correlación precio-nutrientes de cortes comerciales de tambaqui (Colossoma macropomum) y pirarucu (Arapaima gigas) em diferentes clases de peso corporal (Amazonía: Brasil). Research, Society and Development, 10(1). https://doi.org/10.33448/rsd-v10i1.11698

Da Silva, G. C. D. O., Sousa, S., Carvalho, J. W. P., Guedes, S. F., \& Loss, R. A. (2018). Obtenção e caracterização físico-química e microbiológica da gelatina de resíduos de matrinxã (Brycon amazonicus) e tambaqui (Colossoma macroponum). Acta of Fisheries and Aquatic Resources, 6(1), 74-84.

De Faria, R. H. S. A., de Souza, M. L. R., Wagner, P. M., Povh, J. A., \& Ribeiro, R. P. (2003). Rendimento do processamento da tilápia do Nilo (Oreochromis niloticus Linnaeus, 1757) e do pacu (Piaractus mesopotamicus Holmberg, 1887. Acta Scientiarum. Animal Sciences, 25(1), 21-24. https://doi.org/10.4025/actascianimsci.v25i1.2068.

De Lima, L. K. F. (2013). Reaproveitamento de resíduos sólidos na cadeia agroindustrial do pescado. Embrapa Pesca e Aquicultura-Documentos (INFOTECA-E).

De Lima, L. K. F., dos Santos Noleto, S., dos Santos, V. R. V., de Bem Luiz, D., \& Kirschnik, P. G. (2018). Rendimento e composição centesimal do tambaqui (Colossoma macropomum) por diferentes cortes e categorias de peso. Revista Brasileira de Higiene e Sanidade Animal: RBHSA, 12 (2), 223-235.

De Lima, J. L., Gomes, F. A., Barreto, L. V., Rosa, B. L., de Souza, L. P., Ferreira, J. B., \& de Freitas, H. J. (2020). Silagem ácida e biológica de resíduos de peixes produzidos na Amazônia ocidental-Acre. Brazilian Journal of Development, 6(6), 36677-36693. https://doi.org/10.34117/bjdv6n6-271.

De Sá Leitão, B. R. G., \& Favacho, M. C. (2015). Elaboração e avaliação nutricional da farinha da pele do Tambaqui (Colossoma macropomum) e utilização em produtos alimentícios. Nexus-Revista de Extensão do IFAM, 1(2).

De Souza, M. L. R., Marengoni, N. G., Pinto, A. A., \& da Costa Caçador, W. (2000). Rendimento do processamento da tilápia-do-nilo (Oreochromis niloticus): tipos de corte da cabeça em duas categorias de peso. Acta Scientiarum. Animal Sciences, $22,701-706$. https://doi.org/10.4025/actascianimsci.v22i0.2926

Dos Santos Nascimento, M., Pereira, S. J. B., dos Santos, R. F., \& Vieira, A. M. (2018). Avaliação e caracterização do processo de compostagem de resíduos de peixes. PUBVET, 12, 133. https://doi.org/10.31533/pubvet.v12n11a217.1-7.

FAO, F. (2016). Agriculture Organization of the United Nations. The state of world fisheries and aquaculture. In Meeting the Sustainable Development Goals.

Fernandes, T. R. C., da Costa Doria, C. R., \& Menezes, J. T. B. (2018). Características de carcaça e parâmetros de desempenho do tambaqui (Colossoma macropomum, Cuvier, 1818) em diferentes tempos de cultivo e alimentado com rações comerciais. Boletim do Instituto de Pesca, 36(1), 45-52.

Franco, M. L. R. S., Franco, N. P., Gasparino, E., Dorado, D. M., Prado, M., \& Vesco, A. P. D. (2013). Comparação das peles de tilápia do Nilo, pacu e tambaqui: Histologia, composição e resistência. Archivos de zootecnia, 62(237), 21-32. http://dx.doi.org/10.4321/S0004-05922013000100003.

Gomiero, J. S. G., Ribeiro, P. A. P., Ferreira, M. W., \& Logato, P. V. R. (2003). Rendimento de carcaça de peixe matrinxã (Brycon cephalus) nos diferentes cortes de cabeça. Ciência e Agrotecnologia, 27(1), 211-216. https://doi.org/10.1590/S1413-70542003000100027.

Guimarães, C. C., Silva, A. J. I., Cruz, F. G. G., Rufino, J. P. F., Silva, A. F., \& Costa, V. R. (2019). Digestibility and Physicochemical Characteristics of Tambaqui Waste Biological Silage Meal Included in Commercial Layer Diets. Brazilian Journal of Poultry Science, 21(3). https://doi.org/10.1590/1806-90612018-0869.

IBGE (2018). Produção da pecuária municipal 2018. Instituto Brasileiro de Geografia e Estatística-IBGE, 46, 1-8. www.biblioteca.ibge.gov.br/visualizacao/periodicos/84/ppm_2018_v46_br_informativo.pdf.

Izel, A. C. U., \& Melo, L. A. S. (2004). Criação de tambaqui (Colossoma macropomum) em tanques escavados no Estado do Amazonas. Embrapa Amazônia Ocidental-Documentos (INFOTECA-E). 
Research, Society and Development, v. 10, n. 4, e13210413849, 2021

(CC BY 4.0) | ISSN 2525-3409 | DOI: http://dx.doi.org/10.33448/rsd-v10i4.13849

Lima, M. D. M., Mujica, P. I. C., \& Lima, A. M. (2012). Caracterização química e avaliação do rendimento em filés de caranha (Piaractus mesopotamicus). Brazilian Journal of Food Technology, 15(SPE), 41-46. https://doi.org/10.1590/S1981-67232012005000031.

Matiucci, M. A., Santos, I. C. dos., Oliveira, G. G., Araújo, E. dos S., Corrêa, S. S., Ribeiro, R. P., Souza, M. L. R. de., \& Feihrmann, A. C. (2021). Aproveitamento de resíduos da filetagem de tilápia na produção de patê com adição de óleo essencial de orégano. Research, Society and Development, 10(2), e59510213059. https://doi.org/10.33448/rsd-v10i2.13059.

Mello, F. D., Oliveira, C. A., Ribeiro, R. P., Resende, E. K., Povh, J. A., Fornari, D. C., ... \& Streit Jr, D. (2015). Growth curve by Gompertz nonlinear regression model in female and males in tambaqui (Colossoma macropomum). Anais da Academia Brasileira de Ciências, 87(4), 2309-2315. http://dx.doi.org/10.1590/0001-3765201520140315.

Nascimento, M., Freitas, K. F., \& Da Silva, M. V. (2014). Produção e caracterização de silagens de resíduos de peixes comercializados no mercado público de Parnaíba-PI. Enciclopédia biosfera, 10(18), 2450-2458.

Nóbrega, T. C., de Souza, L. A., Bezerra, S. C., \& Lobo, B. P. (2020). Variação temporal e origem do Tambaqui (Colossoma macropomum) desembarcado na feira da panair. Brazilian Journal of Development, 6(2), 9118-9132. https://doi.org/10.34117/bjdv6n2-283.

Pedroza Filho, M. X., Barroso, R. M., \& Flores, R. M. V. (2014). Diagnóstico da cadeia produtiva da piscicultura no Estado de Tocantins. Embrapa Pesca e Aquicultura-Boletim de Pesquisa e Desenvolvimento (INFOTECA-E).

Rosa, L. P., dos Santos, C. M., Barros, L.S., Peixoto, T. S. R., Gonçalves, K. C., Cruz, L. C. C. \& Cruz, P. A. (2018). Mathematical modeling of tambaqui growth pattern through the gompertz model. Revista Brasileira de Engenharia de Pesca. Eng. Pesca, 11(2).

Souza, M. D., Lima, S., \& Vargas, L. (2001). Industrialização, comercialização e perspectivas. Moreira, HLM; Vargas, L.; Ribeiro, RP; Zimmermann, S. Fundamentos da moderna aquicultura. Canoas: ULBRA, 149-181.

Souza, M. L. R. D. (2002). Comparação de seis métodos de filetagem, em relação ao rendimento de filé e de subprodutos do processamento da Tilápia-do-Nilo (Oreochromis niloticus). Revista Brasileira de Zootecnia, 31(3), 1076-1084. https://doi.org/10.1590/S1516-35982002000500003.

Souza, M. L. R. D., Macedo-Viegas, E. M., \& Kronka, S. D. N. (1999). Influência do método de filetagem e categorias de peso sobre rendimento de carcaça, filé e pele da tilápia do Nilo (Oreochromis niloticus). Revista Brasileira de Zootecnia, 28(1), 1-6. https://doi.org/10.1590/S1516-35981999000100001.

Valente, B. S., Xavier, E. G., Pereira, H. D. S., \& Pilotto, M. V. T. (2016). Compostagem de resíduos da filetagem de pescado marinho e casca de arroz. Revista Brasileira de Saúde e Produção Animal, 17(2), 237-248. https://doi.org/10.1590/S1519-99402016000200011. 